\title{
Axionlike Particles from Hypernovae
}

\author{
Andrea Caputo®, ${ }^{1,2,3}$ Pierluca Carenza $\odot{ }^{4,5}$ Giuseppe Lucente $\odot,{ }^{4,5}$ Edoardo Vitagliano $\odot,{ }^{6}$ \\ Maurizio Giannotti $\odot,{ }^{7}$ Kei Kotake, ${ }^{8}$ Takami Kuroda $\odot,{ }^{9}$ and Alessandro Mirizzi ${ }^{4,5}$ \\ ${ }^{1}$ School of Physics and Astronomy, Tel-Aviv University, Tel-Aviv 69978, Israel \\ ${ }^{2}$ Department of Particle Physics and Astrophysics, Weizmann Institute of Science, Rehovot 7610001, Israel \\ ${ }^{3}$ Max-Planck-Institut für Physik (Werner-Heisenberg-Institut), Föhringer Ring 6, 80805 München, Germany \\ ${ }^{4}$ Dipartimento Interateneo di Fisica "Michelangelo Merlin," Via Amendola 173, 70126 Bari, Italy \\ ${ }^{5}$ Istituto Nazionale di Fisica Nucleare-Sezione di Bari, Via Orabona 4, 70126 Bari, Italy \\ ${ }^{6}$ Department of Physics and Astronomy, University of California, Los Angeles, California 90095-1547, USA \\ ${ }^{7}$ Physical Sciences, Barry University, 11300 NE 2nd Avenue, Miami Shores, Florida 33161, USA \\ ${ }^{8}$ Department of Applied Physics and Research Institute of Stellar Explosive Phenomena, Fukuoka University, \\ Fukuoka 814-0180, Japan \\ ${ }^{9}$ Max-Planck-Institut für Gravitationsphysik, Am Mühlenberg 1, D-14476 Potsdam-Golm, Germany
}

(Received 22 April 2021; revised 20 July 2021; accepted 30 September 2021; published 26 October 2021)

It was recently pointed out that very energetic subclasses of supernovae ( $\mathrm{SNe}$ ), like hypernovae and superluminous SNe, might host ultrastrong magnetic fields in their core. Such fields may catalyze the production of feebly interacting particles, changing the predicted emission rates. Here we consider the case of axionlike particles (ALPs) and show that the predicted large scale magnetic fields in the core contribute significantly to the ALP production, via a coherent conversion of thermal photons. Using recent state-ofthe-art supernova (SN) simulations, including magnetohydrodynamics, we find that, if ALPs have masses $m_{a} \sim \mathcal{O}(10) \mathrm{MeV}$, their emissivity in such rare but exciting conditions via magnetic conversions would be over 2 orders of magnitude larger than previously estimated. Moreover, the radiative decay of these massive ALPs would lead to a peculiar delay in the arrival times of the daughter photons. Therefore, high-statistics gamma-ray satellites can potentially discover MeV ALPs in an unprobed region of the parameter space and shed light on the magnetohydrodynamical nature of the SN explosion.

DOI: 10.1103/PhysRevLett.127.181102

Introduction.-Core collapse supernovae ( $\mathrm{SNe})$ are recognized as extremely efficient laboratories for light, feebly interacting particles, such as neutrinos, axionlike particles (ALPs), and dark photons [1,2]. Very often, supernova (SN) bounds significantly constrain the viable parameter space for these particles, providing guidance for their experimental searches. The enthusiasm for $\mathrm{SNe}$ as laboratories for astroparticle physics has grown in recent years, thanks to advancements in numerical simulations and the development of new neutrino detectors, which might provide important information about both the explosion mechanism of $\mathrm{SNe}$ and fundamental physics (see, e.g., Refs. [3-5] for recent reviews).

The current paradigm of the SN explosion mechanism is based on the neutrino-driven scenario, in which neutrino energy deposition revitalizes the stalled shock wave (see, e.g., Refs. [6,7]). However, there are some very

Published by the American Physical Society under the terms of the Creative Commons Attribution 4.0 International license. Further distribution of this work must maintain attribution to the author(s) and the published article's title, journal citation, and DOI. Funded by SCOAP ${ }^{3}$. energetic subclasses of supernovae, including hypernovae and superluminous $\mathrm{SNe}$ [8], which are highly unlikely to be explained by the conventional neutrino-driven explosion. Hypernovae and superluminous $\mathrm{SNe}$ are fascinating and rare events, with a typical Galactic rate $\sim 10^{-5} \mathrm{yr}^{-1}$ [9], often advocated as an explanation of astrophysical conundrums such as $r$-process nucleosynthesis [10] or the source of Galactic positrons [11]. The most plausible scenario to account for these extreme events requires additional energy injection via the magnetohydrodynamically driven (MHD) explosions [12]. This situation has been explored in very recent dedicated numerical studies $[13,14]$, finding that in this case the $\mathrm{SN}$ core might host ultrastrong magnetic fields $\left(B \gtrsim 10^{15} \mathrm{G}\right.$ [15]). The presence of a strong magnetic field is not the only possibility for such a subclass of SNe. For example, instead of considering a very energetic explosion, one may actually consider the possibility that the brightness of these events is due to the interaction of the ejecta with an extended and dense circumstellar envelope [16]. Nevertheless, the presence of strong magnetic field remains the most plausible scenario. Furthermore, such strong magnetic fields are also a plausible origin for more common types 
of SNe, like type Ic-bl SNe [15], for which the analysis of the present Letter applies as well.

In light of these recent developments, it becomes essential to consider the question of whether the presence of potentially very large magnetic fields might influence significantly the emission of light, feebly interacting particles from SNe. It is known, for example, that if neutrinos possess a magnetic moment $\mu \gtrsim 10^{-13} \mu_{B}$, where $\mu_{B}$ is the Bohr magneton, strong magnetic fields may affect their flavor conversions in the SN core, leading to peculiar observational signatures (e.g., $[17,18])$.

However, the impact on other feebly interacting particles has never been studied before. Here we consider ALPs, which are among the most prominent and well-studied new physics candidates [5,19], and show that the presence of a magnetic field may have a significant impact on the emission of these particles if their mass falls in the $m_{a} \sim$ $\mathcal{O}(10) \mathrm{MeV}$ mass range. Our conclusions apply to a rare but spectacular class of events. As such, observations in the near future seem challenging and unlikely, but they would represent a completely new window on axion physics, SN explosion mechanisms, magnetic fields, early Universe cosmology, and much more.

Axionlike particles and supernovae.-ALPs are pseudoscalar bosons $a$ with a two-photon vertex, described by the Lagrangian [20]

$$
\mathcal{L}_{a \gamma}=-\frac{1}{4} g_{a \gamma} F_{\mu \nu} \tilde{F}^{\mu \nu} a,
$$

where $g_{a \gamma}$ is the effective ALP-photon vertex, $F_{\mu \nu}$ is the electromagnetic field, and $\tilde{F}^{\mu \nu}$ is its dual. These particles emerge in different extensions of the standard model (see Refs. [5,21] for recent reviews) and are the object of an intense experimental investigation [22,23]. Furthermore, astrophysical observations on different energy scales offer valuable opportunities to search for these particles [24-26].

Core-collapse $\mathrm{SNe}$ are particularly powerful cosmic factories for ALPs [2,24]. Notably, the SN 1987A neutrino detection has been a milestone event also for axion physics [27-29]. The dominant production channel in SNe for light ALPs coupling exclusively to photons is the Primakoff emission [30], in which thermal photons are converted into ALPs in the electrostatic field of protons. Once produced, SN ALPs provide different signatures depending on their mass. Very light ALPs $\left(m_{a} \lesssim 10^{-10} \mathrm{eV}\right)$ leave the star and convert into gamma rays in the magnetic field of the Milky Way [31,32]. Indeed, the lack of a gamma-ray signal in the Gamma-Ray Spectrometer on the Solar Maximum Mission in coincidence with the observation of the neutrinos emitted from SN 1987A provides a strong bound on the ALP coupling to photons $[31,32]$. The most recent analysis finds $g_{a \gamma}<5.3 \times 10^{-12} \mathrm{GeV}^{-1}$, for $m_{a}<$ $4 \times 10^{-10} \mathrm{eV}$ [33]. On the other hand, ALPs with $m_{a} \sim$ $\mathcal{O}(0.1-100) \mathrm{MeV}$ decay into photons, thus producing a gamma-ray flux without the need for a galactic magnetic field. In this case, a similar analysis gives $g_{a \gamma} \lesssim$ $10^{-11} \mathrm{GeV}^{-1}$ at $m_{a} \simeq 10 \mathrm{MeV}$ [34]. Intriguing opportunities to sharpen these bounds are offered by the detection of an ALP burst in future (extra)galactic SN explosions $[35,36]$ or from the diffuse ALP flux from all past corecollapse SNe in the Universe [37]. If detected, the ALP flux would carry information on the deepest SN regions, complementary to the ones provided by neutrinos and gravitational waves [38,39].

Magnetic fields could play a significant role in the ALP production itself. ALPs may be produced by the scattering of photons on an external macroscopic magnetic field, rather than on the electrostatic field of charged particles as in Primakoff emission. Recent investigations have shown, for example, that the magnetic ALP production in the Sun may dominate over the Primakoff production, particularly in some energy ranges [40-42]. An equivalent analysis for the $\mathrm{SNe}$ is lacking because of the absence, until very recently, of numerical SNe simulations that also included a magnetic field. In this Letter, we fill this gap and investigate the role that ultrastrong magnetic fields in a SN core have in the emissivity of ALPs.

MHD supernova model.-For this purpose, we consider a fully relativistic 3D magnetorotational core-collapse SN simulation with multienergy neutrino transport [43]. The progenitor model is a $20 M_{\odot}$ star with solar metallicity from Ref. [44], while the nuclear part is described by the equation of state SFHo [45]. Since the original progenitor model assumed neither rotation nor magnetic field during its evolution, a cylindrical rotation and a dipolarlike magnetic field was added in Ref. [43]. The resulting star has a central initial angular frequency and magnetic field strength of $1 \mathrm{rad} \mathrm{s}^{-1}$ and $10^{12} \mathrm{G}$, respectively. These lead to a dynamically relevant magnetic field of $B \gtrsim 10^{15} \mathrm{G}$ inside the protoneutron star after bounce through the magnetic compression and winding effects. This model experiences the so-called magnetorotational explosion soon after bounce and the shock wave reaches $\sim 4000 \mathrm{~km}$ from the center at the final simulation postbounce time $t_{\mathrm{pb}}=370 \mathrm{~ms}$.

In Fig. 1, we show the angle-averaged radial profile of the magnetic field strength $B$ (dashed red) at $t_{\mathrm{pb}}=370 \mathrm{~ms}$ in the $\mathrm{SN}$ core $(r \lesssim 50 \mathrm{~km})$ for the $\mathrm{SN}$ model we are considering. In the very inner core $r \lesssim 5 \mathrm{~km}$ the magnetic field assumes a constant value $B \simeq 4 \times 10^{15} \mathrm{G}$. It reaches its maximum $B_{\max } \simeq 7 \times 10^{15} \mathrm{G}$ at $r \simeq 10 \mathrm{~km}$, where the strong shear motion appears, while it decreases at larger radii, becoming smaller than $10^{15} \mathrm{G}$ at $r \gtrsim 30 \mathrm{~km}$.

We also show the angle-averaged radial profiles for the temperature $T$ (blue), plasma frequency $\omega_{\mathrm{pl}}$ (orange), and screening scale $\kappa_{s}$ (black) in the inner SN core. The plasma frequency, defined as $\omega_{\mathrm{pl}} \simeq 16.3 \mathrm{MeV}\left(Y_{e} \rho_{14}\right)^{1 / 3}$ [46], where $\rho_{14}=\rho / 10^{14} \mathrm{~g} \mathrm{~cm}^{-3}$ and $Y_{e}$ is the electron 


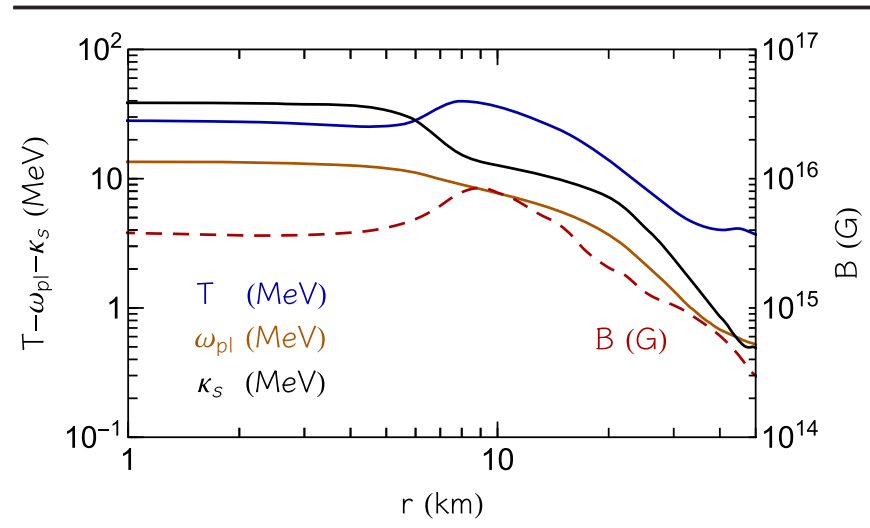

FIG. 1. Angle-averaged radial profile for the temperature $T$ (blue), plasma frequency $\omega_{\mathrm{pl}}$ (orange), screening scale $\kappa_{s}$ (black), and magnetic field strength $B$ (dashed red) at $t_{\mathrm{pb}}=370 \mathrm{~ms}$.

fraction, plays the role of an "effective photon mass." The screening scale $\kappa_{s}$ accounts for the correlation effects of the charged particles in the stellar medium. Since protons are only partially degenerate, a good estimate of the screening scale is given by $\kappa_{s}^{2}=4 \pi \alpha n / T$, with $n$ being the proton number density (see discussion in Ref. [33] for the effects of partial degeneracy).

ALP Primakoff emission.-The main ALP production channel in a SN core for ALPs with masses $m_{a} \lesssim 80 \mathrm{MeV}$ is the Primakoff process. Since electrons are highly degenerate in the SN core, their phase space is Pauli blocked and their contribution to the ALP production is negligible. Therefore, the most substantial contribution to the Primakoff process comes from free protons, which are only partially degenerate in the $\mathrm{SN}$ core. With these assumptions, the Primakoff production rate for massive ALPs is $[29,47]$

$$
\begin{aligned}
\Gamma_{P}= & 2 g_{a r}^{2} \frac{T \kappa_{s}^{2}}{32 \pi} \frac{p}{E}\left\{\frac{\left[(k+p)^{2}+\kappa_{s}^{2}\right]\left[(k-p)^{2}+\kappa_{s}^{2}\right]}{4 k p \kappa_{s}^{2}}\right. \\
& \left.\times \ln \left[\frac{(k+p)^{2}+\kappa_{s}^{2}}{(k-p)^{2}+\kappa_{s}^{2}}\right]-\frac{\left(k^{2}-p^{2}\right)^{2}}{4 k p \kappa_{s}^{2}} \ln \left[\frac{(k+p)^{2}}{(k-p)^{2}}\right]-1\right\},
\end{aligned}
$$

where the factor 2 accounts for the photon polarization states, $E$ and $p$ are, respectively, the energy and momentum of the ALP, and $k$ is the momentum of the photon. In the conditions of interest for us, we can neglect the proton recoil energy and take the photon energy equal to the ALP energy, $\omega=E$.

ALP emission via magnetic conversions.-The rate for photon-ALP conversion in a magnetized plasma was recently calculated in Refs. [40-42]. ALPs can be resonantly produced in an external $B$ field when the dispersion relations of longitudinal $(L)$ or transverse $(T)$ photon degrees of freedom match the dispersion relation of the ALP. The resonant emission rate is $[40,42,48]$

$$
\begin{aligned}
\Gamma_{B}= & m_{a}^{2} B_{\|}^{2} g_{a \gamma}^{2} \frac{\pi}{2 \omega^{2}} Z_{L} \delta\left[\omega-\omega_{0}^{L}(k)\right] \\
& +\omega^{2} B_{\perp}^{2} g_{a \gamma}^{2} \frac{\pi}{2 \omega^{2}} Z_{T} \delta\left[\omega-\omega_{0}^{T}(k)\right],
\end{aligned}
$$

where the factor $Z_{L, T}$ can be interpreted as renormalizing the coupling to the axion, $B_{\|}$and $B_{\perp}$ are the $B$ field components parallel and orthogonal to the photon momentum, and $\omega_{0}^{T, L}(k)$ is the dispersion relation for the considered electromagnetic mode [2] (see the Appendix for further details). Any excitation of the electromagnetic field in a medium, both longitudinal and transverse, is called "plasmon."

A word of caution is in order. The presence of very strong magnetic fields, $B \simeq 10^{16} \mathrm{G}$, could potentially affect the electron wave functions and, moreover, generate QED nonlinear effects. However, $\mathrm{SNe}$ feature a strongly degenerate and ultrarelativistic plasma, so the Fermi momentum is much larger than the synchrotron frequency $\omega_{B}=e B / p_{F}$. In such environments, the gas behaves quasiclassically, as the magnetic field does not quantize the electron wave functions [49-51]. This hierarchy also prevents from the production of electron-positron pairs. Moreover, the electron mass is modified to $m_{e}^{2} \simeq$ $\left(e^{2} / 2 \pi^{2}\right) p_{F}^{2} \gg\left(m_{e}^{\mathrm{vac}}\right)^{2}$ [52,53], which implies that the critical magnetic field $B_{c}=m_{e}^{2} / e$ is much larger than in vacuum. Thus, we can neglect also birefringence effects [54,55], which typically inhibit the conversion in intense magnetic fields in vacuum [56]. Therefore, we identify a previously overlooked condition for efficient ALP-photon conversion in exceptionally strong magnetic fields that does not rely on the ALP being nonrelativistic [57].

Comparison of ALP fluxes.-The ALP emissivity for a given process is found by integrating the production rate over the photon thermal spectrum

$$
Q_{a}=\int \frac{d^{3} \mathbf{k}}{(2 \pi)^{3}} \frac{\Gamma \omega}{e^{\omega / T}-1}
$$

and the ALP luminosity by integrating the previous expression over the SN model,

$$
L_{a}=\int d \Omega \int Q_{a}(r, \Omega) r^{2} d r=\int d \omega \omega \frac{d N_{a}}{d \omega d t},
$$

where $d N_{a} / d \omega d t$ is the differential ALP production rate. The latter is shown in Fig. 2 for three different values of the ALP mass, $m_{a}=2,5$, and $13 \mathrm{MeV}$. The plasmon-ALP conversion clearly dominates over Primakoff, especially for energies $\omega \lesssim 200 \mathrm{MeV}$, if the ALP mass is in the range $4 \lesssim m_{a} \lesssim 14 \mathrm{MeV}$. For $m_{a} \lesssim 4 \mathrm{MeV}$, the resonance conversion takes place in the external regions of the star, where the magnetic field is too weak to lead to a sizable production. On the other hand, if the mass is too large, $m_{a} \gg 14 \mathrm{MeV}$, the conversion happens in the very inner 


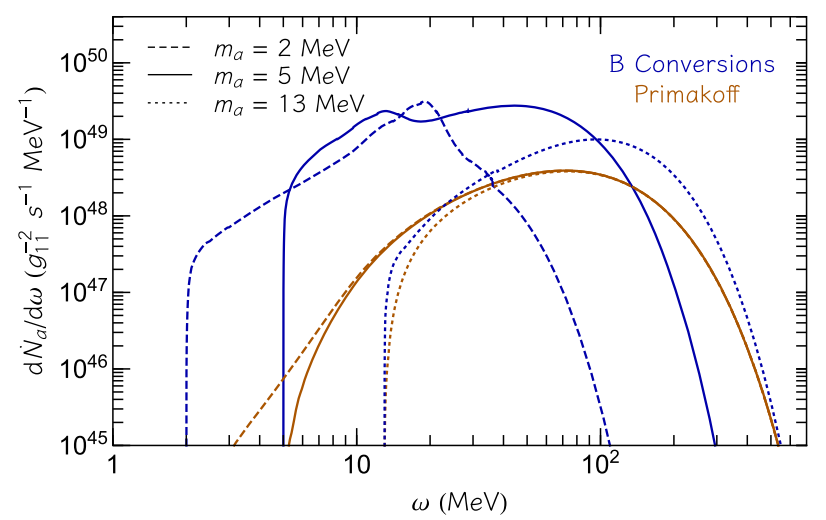

FIG. 2. Differential ALP production rate for the Primakoff (orange) and the $B$-conversion (blue) processes, for $m_{a}=2,5$, $13 \mathrm{MeV}$ (dashed, solid, and dotted lines, respectively) and $g_{a \gamma}=10^{-11} \mathrm{GeV}^{-1}$, at $t_{\mathrm{pb}}=370 \mathrm{~ms}$. In the case of $m_{a}=2$, $5 \mathrm{MeV}$, at $\omega \gtrsim 10 \mathrm{MeV}$, we notice a small peak due to the presence — in a very narrow energy range — of longitudinal modes conversion.

core $(r \lesssim 5 \mathrm{~km})$, where the plasmon population is suppressed. Notice that the contribution of the $L$ mode is relevant only in a very narrow energy range, at low energy. At large energies, which — as we shall see below-are the most interesting for a possible detection, the transverse are the only relevant modes. Integrating the differential flux gives the total luminosity. We find that the $B$-conversion luminosity is larger than the Primakoff luminosity, $L_{a, B}>$ $L_{a, P}$, for $4 \lesssim m_{a} \lesssim 14 \mathrm{MeV}$, with a peak luminosity $L_{a, B}^{\max } \simeq$ $10^{2} L_{a, P} \simeq 10^{49}\left(g_{a \gamma} / 10^{-11} \mathrm{GeV}^{-1}\right)^{2} \mathrm{ergs}^{-1}$ for $m_{a} \simeq 10 \mathrm{MeV}$.

After being produced in the SN core, ALPs with $\mathcal{O}(1) \mathrm{MeV}$ mass can decay into two photons with energy $E_{\gamma}=\omega / 2$, producing a large gamma-ray flux that can be observed with existent [58] and planned [59-61] gammaray detectors. This flux will feature a characteristic time distribution, as the distance traveled by the ALP before decaying plus the distance traveled by daughter photons is larger than the distance of the SN from Earth.

Following Refs. [34,62], we estimate the time delay of an ALP-originated photon arriving at Earth, with respect to a massless particle coming directly from the $\mathrm{SN}$, as

$$
t=\frac{L_{1}}{\beta}+L_{2}-d_{\mathrm{SN}}
$$

where $\beta$ is the ALP velocity, $L_{1}=64 \omega \pi /\left(g_{a \gamma}^{2} m_{a}^{4}\right) \times$ $\sqrt{1-\left(m_{a} / \omega\right)^{2}}$ is the ALP decay length, and $L_{2}=-L_{1} \cos \alpha+\sqrt{d_{\mathrm{SN}}^{2}-L_{1}^{2} \sin ^{2} \alpha}$, with $\alpha$ as the angle between the photon and the ALP momentum, is the distance covered by the daughter photon before reaching Earth. In the relativistic limit, $\sin \alpha \simeq\left(\omega / m_{a}\right)^{-1}$ [34]. Finally, $d_{\mathrm{SN}}$ is the Earth-SN distance, which we fix to $d_{\mathrm{SN}}=10 \mathrm{kpc}$ and, in all cases of interest for us, is much larger than $L_{1}$.
The flux of daughter photons can be observed over the interval of several days with gamma-ray instruments such as the Fermi Large Area Telescope (LAT) [58]. In general, the number of events per unit time from the daughter photons expected in a gamma-ray detector with a delay time $\tau$ can be estimated as

$$
\begin{aligned}
\dot{N}_{\gamma}(\tau)= & \frac{2}{4 \pi d_{\mathrm{SN}}^{2}}\left(\frac{d t\left(\omega_{\tau}^{*}\right)}{d \omega}\right)^{-1} \frac{d N_{a}\left(\omega_{\tau}^{*}\right)}{d \omega} A\left(\omega_{\tau}^{*} / 2\right) \\
& \times e^{-R^{*} / L_{1}}\left(1-e^{-d_{\mathrm{SN}} / L_{1}}\right),
\end{aligned}
$$

where $d N_{a} / d \omega$ is the SN ALP production rate, $d t / d \omega$ is obtained from Eq. (6), $R^{*}=10^{14} \mathrm{~cm}$ [63] is the effective $\mathrm{SN}$ radius, and the factor $e^{-R^{*} / L_{1}}$ ensures that only ALPs decaying outside the SN are counted. Analogously, the factor $\left(1-e^{-d_{\mathrm{SN}} / L_{1}}\right)$ selects ALPs decaying before reaching Earth, and $A(\omega)$ is the detector effective area. Notice that Eq. (7) should be evaluated at $\omega=\omega_{\tau}^{*}$, where the ALP energy $\omega_{\tau}^{*}$ is obtained by solving Eq. (6) with $t=\tau$.

Here, to give a realistic assessment of the potential to detect photons from ALP decay, we consider the specific case of Fermi LAT with Pass 8 event selection [64,65]. In Fig. 3, we show the number of expected events per unit time $\dot{N}_{\gamma}$-with $2 \sigma$ Poissonian fluctuation contours-as a function of the time delay $\tau$. The blue band refers to photons from the decay of Primakoff ALPs, and the orange band refers to photons from ALPs produced in the SN magnetic field. In both cases, we are fixing the ALP mass to $m_{a}=5 \mathrm{MeV}$ and the ALP-photon coupling to $g_{a \gamma}=10^{-11} \mathrm{GeV}^{-1}$.

Though the two signals have a qualitatively similar time evolution, the difference between them is manifest. Since ALPs produced through magnetic conversion are considerably less energetic than Primakoff ALPs, their daughter photons arrive with a more substantial delay. Thus, the photon flux in the first couple of days, being dominated by Primakoff ALPs, has a very weak dependence on the magnetic field, and would allow one to fix the ALP coupling to photons. The photon flux of the following days, after the Primakoff contribution decline, could then provide information about the intensity of the magnetic field. Therefore, reconstructing the time evolution of the signal allows us to gain confidence about the presence of a strong magnetic field in the $\mathrm{SN}$ and to measure its intensity.

As a final remark, we underline that the parameter region we are probing can also be constrained from cosmological observations based on big-bang nucleosynthesis. The cosmological argument, however, can be (at least partially) circumvented in low-reheating cosmological scenarios [66]. Therefore, a SN ALP detection in the mass range probed in this Letter would have important implications also for cosmology, pointing toward a nonstandard cosmological scenario. This shows a nice synergy between astrophysical and cosmological arguments to probe ALPs. 


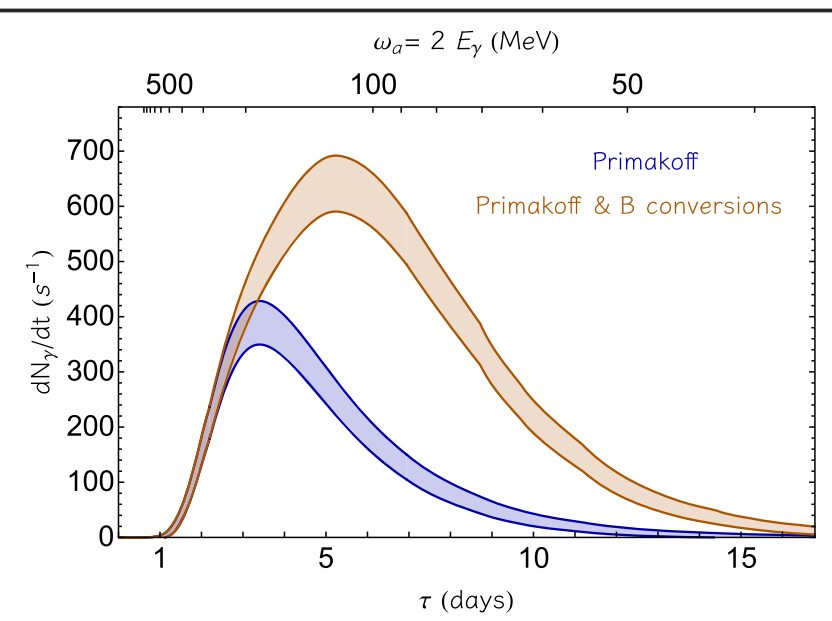

FIG. 3. Number of events per unit time with $2 \sigma$ Poissonian fluctuation contours expected to be observed by Fermi LAT, as a function of the time delay, obtained considering only Primakoff (blue), and both Primakoff and $B$ conversions (orange), for $m_{a}=$ $5 \mathrm{MeV}$ and $g_{a \gamma}=10^{-11} \mathrm{GeV}^{-1}$. On the upper $x$ axis, the ALP energy corresponding to each delay time $\tau$ is shown.

Conclusions.-Very energetic supernovae, such as hypernovae and superluminous $\mathrm{SNe}$, host intense magnetic fields in their cores. We have shown that these fields would trigger a resonant ALP production via conversion of photons. Furthermore, we have demonstrated that if ALPs have a mass in the range $4 \lesssim m_{a} \lesssim 14 \mathrm{MeV}$, the $B$ conversion would overcome the Primakoff production by over an order of magnitude, becoming the dominant ALP production mechanism in $\mathrm{SNe}$ with $B \sim \mathcal{O}\left(10^{15}\right) \mathrm{G}$.

These massive ALPs, with $m_{a} \sim \mathcal{O}(10) \mathrm{MeV}$ and $g_{a \gamma} \sim 10^{-11} \mathrm{GeV}^{-1}$, have a decay length much shorter than the SN distance and so would produce an observable photon flux on Earth. Depending on the axion coupling, the flux might be observable by gamma-ray instruments such as Fermi LAT for well over a week. Furthermore, the temporal distribution of the flux is peculiar of the production mechanisms, providing a transparent way to recognize if ALPs are produced through conversions in a magnetic field or through the Primakoff process. Indeed, standard signals associated with violent events such as hypernovae and superluminous $\mathrm{SNe}$ are expected to peak at larger energies, above the $\mathrm{GeV}$ [67] and have a short duration. For this reason, hypernovae and superluminous $\mathrm{SNe}$ are often advocated as an explanation for gamma-ray bursts, which are extremely powerful signals that last from seconds to minutes [68]. Finally, since the magnetic production mechanism is very sensitive to the ALP mass, an accurate analysis of the time delay would allow us to infer $m_{a}$.

The discovery of a gamma-ray flux from a Galactic SN with the temporal properties described in this Letter would have enormous consequences both for ALP and SN physics. On the one hand, it would point out the existence of ALPs and allow us to pin down its mass; on the other, it would be the smoking gun for the presence of ultrastrong magnetic fields in the SN core. Furthermore, the signal from axion decay will also be spread in angles away from the line of sight [34]; this may be particularly relevant in the future for closer $\mathrm{SNe}$ and gamma-ray instruments with better angular resolution.

We stress once more that our study mainly applies to a rare class of events. As such, observations in the near future seem unlikely but would offer an exciting possibility to learn about axions, as well as high-energy astrophysics and early Universe cosmology. This confirms once more $\mathrm{SNe}$ as multimessenger laboratories for particle physics and shows how much could be learned about $\mathrm{SNe}$ from the detection of ALP-induced processes.

We warmly thank Niccolò Di Lalla, Georg Raffelt, and Jin Matsumoto for useful discussions. A. C. is supported by the Foreign Postdoctoral Fellowship Program of the Israel Academy of Sciences and Humanities. A. C. acknowledges support from the Israel Science Foundation (Grant No. 1302/19), the US-Israeli BSF (Grant No. 2018236), and the German Israeli GIF (Grant No. I-2524-303.7). A. C. acknowledges hospitality of the Max Planck Institute for Physics in Munich where part of this work was carried out. The work of P. C., G. L., and A. M. is partially supported by the Italian Istituto Nazionale di Fisica Nucleare (INFN) through the "Theoretical Astroparticle Physics" project and by the research Grant No. 2017W4HA7S "NAT-NET: Neutrino and Astroparticle Theory Network" under the program PRIN 2017 funded by the Italian Ministero dell'Università e della Ricerca (MUR). The work of E. V. was supported in part by the U.S. Department of Energy (DOE) Award No. DE-SC0009937. K. K. is supported by the Grant-in-Aid for the Scientific Research of Japan Society for the Promotion of Science (JSPS) KAKENHI (Grants No. JP17H01130, No. JP17H06364, and No. JP17H06357), and by the Central Research Institute of Explosive Stellar Phenomena (REISEP) of Fukuoka University and the associated project (No. 207002).

Appendix: Plasmon dispersions and renormalization factors.-In this Appendix, we recollect the dispersion relations for the electromagnetic excitations in a QED plasma [2]. For an isotropic medium, in the Lorenz gauge, these dispersion relations for the longitudinal and transverse modes read

$$
\begin{gathered}
\sqrt{\omega^{2}-v_{*}^{2} k^{2}}=\omega_{0}^{L}(k) \equiv \sqrt{\omega_{p}^{2}\left[1-G\left(v_{*}^{2} k^{2} / \omega^{2}\right)\right]}, \\
\sqrt{\omega^{2}-k^{2}}=\omega_{0}^{T}(k) \equiv \sqrt{\omega_{p}^{2}\left[1+1 / 2 G\left(v_{*}^{2} k^{2} / \omega^{2}\right)\right]},
\end{gathered}
$$

where $G$ is an auxiliary function, defined as $G(x)=$ $(3 / x)\{1-(2 x / 3)-[(1-x) / 2 \sqrt{x}] \ln [(1+\sqrt{x}) /(1-\sqrt{x})]\}$, and $v_{*}$ is the typical velocity for the electron and positron in 
the plasma [2]. For the SN profile we considered, $v_{*} \gtrsim 0.99$ throughout the entire star.

The renormalization factors are also needed to evaluate Eq. (3),

$$
\begin{gathered}
Z_{L}=\frac{\omega^{2}}{\omega^{2}-k^{2}} \frac{2\left(\omega^{2}-v_{*}^{2} k^{2}\right)}{3 \omega_{p}^{2}-\left(\omega^{2}-v_{*}^{2} k^{2}\right)}, \\
Z_{T}=\frac{2\left(\omega^{2}-v_{*}^{2} k^{2}\right)}{\omega^{2}\left[3 \omega_{p}^{2}-2\left(\omega^{2}-k^{2}\right)\right]+\left(\omega^{2}+k^{2}\right)\left(\omega^{2}-v_{*}^{2} k^{2}\right)} .
\end{gathered}
$$

One finds that $Z_{T}$ is always very close to unity, while $Z_{L}$ can strongly deviate from 1 .

[1] G. G. Raffelt, Particle physics from stars, Annu. Rev. Nucl. Part. Sci. 49, 163 (1999).

[2] G. G. Raffelt, Stars as Laboratories for Fundamental Physics (University of Chicago Press, Chicago, 1996).

[3] A. Mirizzi, I. Tamborra, H.-T. Janka, N. Saviano, K. Scholberg, R. Bollig, L. Hudepohl, and S. Chakraborty, Supernova neutrinos: Production, oscillations and detection, Riv. Nuovo Cimento 39, 1 (2016).

[4] S. Horiuchi and J. P. Kneller, What can be learned from a future supernova neutrino detection?, J. Phys. G 45, 043002 (2018).

[5] P. Agrawal et al., Feebly-interacting particles: FIPs 2020 workshop report, arXiv:2102.12143.

[6] H. T. Janka, T. Melson, and A. Summa, Physics of corecollapse supernovae in three dimensions: A sneak preview, Annu. Rev. Nucl. Part. Sci. 66, 341 (2016).

[7] H. T. Janka, Neutrino-driven explosions, Handbook of Supernova, edited by A. Alsabti and P. Murdin (Springer, New York, 2017), https://doi.org/10.1007/978-3-319-218465_109.

[8] T. J. Moriya, E. I. Sorokina, and R. A. Chevalier, Superluminous supernovae, Space Sci. Rev. 214, 59 (2018).

[9] P. Podsiadlowski, P. A. Mazzali, K. Nomoto, D. Lazzati, and E. Cappellaro, The rates of hypernovae and gamma-ray bursts: Implications for their progenitors, Astrophys. J. Lett. 607, L17 (2004).

[10] D. Yong et al., $r$-process elements from magnetorotational hypernovae, Nature (London) 595, 223 (2021).

[11] S. Schanne, M. Casse, B. Cordier, and J. Paul, Hypernovae as possible sources of Galactic positrons, in Contribution to: 35th COSPAR Scientific Assembly (2004) [arXiv:astro-ph/ 0411454v2].

[12] A. Burrows, L. Dessart, E. Livne, C. D. Ott, and J. Murphy, Simulations of magnetically-driven supernova and hypernova explosions in the context of rapid rotation, Astrophys. J. 664, 416 (2007).

[13] J. Matsumoto, T. Takiwaki, K. Kotake, Y. Asahina, and H.R. Takahashi, 2D numerical study for magnetic field dependence of neutrino-driven core-collapse supernova models, Mon. Not. R. Astron. Soc. 499, 4174 (2020).
[14] M. Obergaulinger and M.-A. Aloy, Magnetorotational core collapse of possible GRB progenitors. III. Threedimensional models, Mon. Not. R. Astron. Soc. 503, 4942 (2021).

[15] P. Mösta, C. D. Ott, D. Radice, L. F. Roberts, E. Schnetter, and R. Haas, A large scale dynamo and magnetoturbulence in rapidly rotating core-collapse supernovae, Nature (London) 528, 376 (2015).

[16] E. Sorokina, S. Blinnikov, K. Nomoto, R. Quimby, and A. Tolstov, Type I superluminous supernovae as explosions inside non-hydrogen circumstellar envelopes, Astrophys. J. 829, 17 (2016).

[17] E. K. Akhmedov and T. Fukuyama, Supernova prompt neutronization neutrinos and neutrino magnetic moments, J. Cosmol. Astropart. Phys. 12 (2003) 007.

[18] S. Abbar, Collective oscillations of Majorana neutrinos in strong magnetic fields and self-induced flavor equilibrium, Phys. Rev. D 101, 103032 (2020).

[19] A. Ringwald, Axions and axion-like particles, in Proceedings of the 49th Rencontres de Moriond on Electroweak Interactions and Unified Theories, La Thuile, Italy, 2014, edited by E. Augé, J. Dumarchez, and J. T. T. Vân (ARISF, Paris, 2014), p. 7.

[20] P. Sikivie, Experimental Tests of the Invisible Axion, Phys. Rev. Lett. 51, 1415 (1983); 52, 695(E) (1984).

[21] L. Di Luzio, M. Giannotti, E. Nardi, and L. Visinelli, The landscape of QCD axion models, Phys. Rep. 870, 1 (2020).

[22] I. G. Irastorza and J. Redondo, New experimental approaches in the search for axion-like particles, Prog. Part. Nucl. Phys. 102, 89 (2018).

[23] P. Sikivie, Invisible axion search methods, Rev. Mod. Phys. 93, 015004 (2021).

[24] G. G. Raffelt, Astrophysical axion bounds, Lect. Notes Phys. 741, 51 (2008).

[25] M. Giannotti, I. G. Irastorza, J. Redondo, A. Ringwald, and K. Saikawa, Stellar recipes for axion hunters, J. Cosmol. Astropart. Phys. 10 (2017) 010.

[26] M. Giannotti, I. Irastorza, J. Redondo, and A. Ringwald, Cool WISPs for stellar cooling excesses, J. Cosmol. Astropart. Phys. 05 (2016) 057.

[27] M. S. Turner, Axions from SN 1987a, Phys. Rev. Lett. 60, 1797 (1988).

[28] A. Burrows, M. S. Turner, and R. P. Brinkmann, Axions and SN 1987a, Phys. Rev. D 39, 1020 (1989).

[29] G. Lucente, P. Carenza, T. Fischer, M. Giannotti, and A. Mirizzi, Heavy axion-like particles and core-collapse supernovae: Constraints and impact on the explosion mechanism, J. Cosmol. Astropart. Phys. 12 (2020) 008.

[30] G. G. Raffelt, Astrophysical axion bounds diminished by screening effects, Phys. Rev. D 33, 897 (1986).

[31] J. A. Grifols, E. Masso, and R. Toldra, Gamma-Rays from SN1987A due to Pseudoscalar Conversion, Phys. Rev. Lett. 77, 2372 (1996).

[32] J. W. Brockway, E. D. Carlson, and G. G. Raffelt, SN1987A gamma-ray limits on the conversion of pseudoscalars, Phys. Lett. B 383, 439 (1996).

[33] A. Payez, C. Evoli, T. Fischer, M. Giannotti, A. Mirizzi, and A. Ringwald, Revisiting the SN1987A gamma-ray limit on ultralight axion-like particles, J. Cosmol. Astropart. Phys. 02 (2015) 006. 
[34] J. Jaeckel, P. C. Malta, and J. Redondo, Decay photons from the axionlike particles burst of type II supernovae, Phys. Rev. D 98, 055032 (2018).

[35] M. Meyer, M. Giannotti, A. Mirizzi, J. Conrad, and M. A. Sánchez-Conde, Fermi Large Area Telescope as a Galactic Supernovae Axionscope, Phys. Rev. Lett. 118, 011103 (2017).

[36] M. Meyer and T. Petrushevska, Search for AxionlikeParticle-Induced Prompt $\gamma$-Ray Emission from Extragalactic Core-Collapse Supernovae with the Fermi Large Area Telescope, Phys. Rev. Lett. 124, 231101 (2020); 125, 119901(E) (2020).

[37] F. Calore, P. Carenza, M. Giannotti, J. Jaeckel, and A. Mirizzi, Bounds on axionlike particles from the diffuse supernova flux, Phys. Rev. D 102, 123005 (2020).

[38] K. Nakamura, S. Horiuchi, M. Tanaka, K. Hayama, T. Takiwaki, and K. Kotake, Multimessenger signals of longterm core-collapse supernova simulations: Synergetic observation strategies, Mon. Not. R. Astron. Soc. 461, 3296 (2016).

[39] S. W. Li, L. F. Roberts, and J. F. Beacom, Exciting prospects for detecting late-time neutrinos from core-collapse supernovae, Phys. Rev. D 103, 023016 (2021).

[40] A. Caputo, A. J. Millar, and E. Vitagliano, Revisiting longitudinal plasmon-axion conversion in external magnetic fields, Phys. Rev. D 101, 123004 (2020).

[41] C. A. O'Hare, A. Caputo, A. J. Millar, and E. Vitagliano, Axion helioscopes as solar magnetometers, Phys. Rev. D 102, 043019 (2020).

[42] E. Guarini, P. Carenza, J. Galan, M. Giannotti, and A. Mirizzi, Production of axionlike particles from photon conversions in large-scale solar magnetic fields, Phys. Rev. D 102, 123024 (2020).

[43] T. Kuroda, Impact of a magnetic field on neutrino-matter interactions in core-collapse supernovae, Astrophys. J. 906, 128 (2021).

[44] S. E. Woosley and A. Heger, Nucleosynthesis and remnants in massive stars of solar metallicity, Phys. Rep. 442, 269 (2007).

[45] A. W. Steiner, M. Hempel, and T. Fischer, Core-collapse supernova equations of state based on neutron star observations, Astrophys. J. 774, 17 (2013).

[46] A. Kopf and G. Raffelt, Photon dispersion in a supernova core, Phys. Rev. D 57, 3235 (1998).

[47] L. Di Lella, A. Pilaftsis, G. Raffelt, and K. Zioutas, Search for solar Kaluza-Klein axions in theories of low scale quantum gravity, Phys. Rev. D 62, 125011 (2000).

[48] N. V. Mikheev, G. Raffelt, and L. A. Vassilevskaya, Axion emission by magnetic field induced conversion of longitudinal plasmons, Phys. Rev. D 58, 055008 (1998).

[49] A. D. Kaminker, K. P. Levenfish, and D. G. Yakovlev, Synchrotron emission of neutrino pairs in neutron stars, Sov. Astron. Lett. 17, 450 (1991), https://ui.adsabs.harvard .edu/abs/1991SvAL...17..450K.

[50] V. G. Bezchastnov, P. Haensel, A. D. Kaminker, and D. G. Yakovlev, Neutrino synchrotron emission from dense magnetized electron gas of neutron stars, arXiv:astro-ph/ 9708181.
[51] D. G. Yakovlev, A. D. Kaminker, O. Y. Gnedin, and P. Haensel, Neutrino emission from neutron stars, Phys. Rep. 354, 1 (2001).

[52] M. L. Bellac, Thermal Field Theory, Cambridge Monographs on Mathematical Physics (Cambridge University Press, Cambridge, England, 2011), https://doi.org/10.1017/ CBO9780511721700.

[53] E. J. Levinson and D. H. Boal, Self-energy corrections to fermions in the presence of a thermal background, Phys. Rev. D 31, 3280 (1985).

[54] W.-Y. Tsai and T. Erber, Propagation of photons in homogeneous magnetic fields: Index of refraction, Phys. Rev. D 12, 1132 (1974).

[55] Note that for $B \gtrsim B_{c}$ the correct equations for vacuum birefringence are given in Ref. [54] and differ from those commonly found in the literature [2], where the implicit assumption is $B \ll B_{c}$.

[56] G. Raffelt and L. Stodolsky, Mixing of the photon with low mass particles, Phys. Rev. D 37, 1237 (1988).

[57] A. Hook, Y. Kahn, B. R. Safdi, and Z. Sun, Radio Signals from Axion Dark Matter Conversion in Neutron Star Magnetospheres, Phys. Rev. Lett. 121, 241102 (2018).

[58] W. B. Atwood et al. (Fermi-LAT Collaboration), The Large Area Telescope on the Fermi Gamma-Ray Space Telescope mission, Astrophys. J. 697, 1071 (2009).

[59] M. Tavani et al. (e-ASTROGAM Collaboration), Science with e-ASTROGAM: A space mission for $\mathrm{MeV}-\mathrm{GeV}$ gamma-ray astrophysics, J. High Energy Astrophys. 19, 1 (2018).

[60] R. Caputo et al. (AMEGO Collaboration), All-Sky Medium Energy Gamma-Ray Observatory: Exploring the extreme multimessenger universe, arXiv:1907.07558.

[61] A. E. Egorov, N. P. Topchiev, A. M. Galper, O. D. Dalkarov, A. A. Leonov, S. I. Suchkov, and Y. T. Yurkin, Dark matter searches by the planned gamma-ray telescope GAMMA400, J. Cosmol. Astropart. Phys. 11 (2020) 049.

[62] M. Giannotti, L. D. Duffy, and R. Nita, New constraints for heavy axion-like particles from supernovae, J. Cosmol. Astropart. Phys. 01 (2011) 015.

[63] W. DeRocco, P. W. Graham, D. Kasen, G. Marques-Tavares, and S. Rajendran, Observable signatures of dark photons from supernovae, J. High Energy Phys. 02 (2019) 171.

[64] P. Bruel, T. H. Burnett, S. W. Digel, G. Johannesson, N. Omodei, and M. Wood (Fermi-LAT Collaboration), FermiLAT improved Pass $\sim 8$ event selection, in Proceedings of the 8th International Fermi Symposium: Celebrating 10 Year of Fermi (2018), p. 10, arXiv:1810.11394.

[65] https://www.slac.stanford.edu/exp/glast/groups/canda/lat_ Performance.htm.

[66] P. F. Depta, M. Hufnagel, and K. Schmidt-Hoberg, Robust cosmological constraints on axion-like particles, J. Cosmol. Astropart. Phys. 05 (2020) 009.

[67] N. Renault-Tinacci, K. Kotera, A. Neronov, and S. Ando, Search for $\gamma$-ray emission from superluminous supernovae with the Fermi-LAT, Astron. Astrophys. 611, A45 (2018).

[68] J. Hjorth et al., A very energetic supernova associated with the gamma-ray burst of 29 March 2003, Nature (London) 423, 847 (2003). 